\title{
A simple strategy for culturing morphologically-conserved rat hypothalamic tanycytes
}

\author{
Pablo Nicolás De Francesco ${ }^{1}$ Daniel Castrogiovanni ${ }^{2} \cdot$ Maia Uriarte $^{1}$. \\ Victoria Frassa $^{1}$ - Francina Agosti $^{3}$ - Jesica Raingo ${ }^{3}$ - Mario Perello ${ }^{1}$
}

Received: 25 August 2016/Revised: 2 March 2017 / Accepted: 6 March 2017

(C) Springer-Verlag Berlin Heidelberg 2017

\begin{abstract}
Hypothalamic tanycytes are specialized bipolar ependymal cells that line the floor of the third ventricle. Given their strategic location, tanycytes are believed to play several key functions including being a selective barrier and controlling the amount of hypothalamic-derived factors reaching the anterior pituitary. The in vitro culture of these cells has proved to be difficult. Here, we report an improved method for the generation of primary cultures of rat hypothalamic tanycytes. Ependymal cultures were derived from tissue dissected out of the median eminence region of 10-day-old rats and cultured in a chemically defined medium containing DMEM:F12, serum albumin, insulin, transferrin and the antibiotic gentamycin. After 7 days in vitro, $\sim 30 \%$ of the cultured cells exhibited morphological features of tanycytes as observed by phase contrast or scanning electron microscopy. Tanycyte-like cells were strongly immuno-reactive for vimentin and dopamine-cAMP-regulated phospho-protein (DARPP-32) and weakly immune-reactive for glial fibrillary acidic protein. Tanycyte-like cells displayed a stable negative
\end{abstract}

Mario Perello

mperello@imbice.gov.ar

1 Laboratory of Neurophysiology of the Multidisciplinary Institute of Cell Biology [IMBICE, dependent of the Argentine Research Council (CONICET) and Scientific Research Commission, Province of Buenos Aires (CIC-PBA)], Calle 526 entre 10 y 11, PO Box 403, La Plata 1900, Buenos Aires, Argentina

2 Cell Culture Facility of the Multidisciplinary Institute of Cell Biology [IMBICE, dependent of the Argentine Research Council (CONICET) and Scientific Research Commission, Province of Buenos Aires (CIC-PBA)], La Plata, Buenos Aires, Argentina

3 Laboratory of Electrophysiology of the Multidisciplinary Institute of Cell Biology [IMBICE, dependent of the Argentine Research Council (CONICET) and Scientific Research Commission, Province of Buenos Aires (CIC-PBA)], La Plata, Buenos Aires, Argentina resting plasma membrane potential and failed to show spiking properties in response to current injections. When exposed to fluorescent beads in the culture medium, tanycyte-like cells exhibited a robust endocytosis. Thus, the present method effectively yields cultures containing tanycyte-like cells that resemble in vivo tanycytes in terms of morphologic features and molecular markers as well as electrical and endocytic activity. To our knowledge, this is the first protocol that allows the culturing of tanycyte-like cells that can be individually identified and that conserve the morphology of tanycytes in their natural physiological environment.

Keywords Cell culture $\cdot$ Ependymal cells $\cdot$ Hypothalamus . Median eminence $\cdot$ Glia

\section{Introduction}

The hypothalamic tanycytes are a specialized type of bipolar ependymal cells that form a unicellular epithelium lining the ventral part of the third ventricle, at the level of the median eminence (ME; Goodman and Hajihosseini 2015; Rodríguez et al. 2005). Based on their localization, the directionality of their processes and the expression of differential markers, four tanycyte subtypes can be recognized (Rodríguez et al. 2005). Tanycytes play important physiological functions. On the one hand, tanycytes form a selective barrier that controls the flow of molecules between the cerebrospinal fluid (CSF) and some hypothalamic nuclei and the ME (Rodríguez et al. 2005, 2010). It has been reported that tanycytes are able to take up circulating factors (i.e., leptin) that have diffused to the external layer of the ME from the fenestrated capillaries, mediating their transport into the CSF (Balland et al. 2014). Tanycytes also seem to facilitate the transport of molecules present in the CSF into specific hypothalamic nuclei by mechanisms 
dependent on transcytosis (Peruzzo et al. 2004; Rodríguez et al. 2005). In addition, tanycytes can modulate the activity of several neuroendocrine axes. For instance, tanycytes affect the thyroid axis activity since they express the ectoenzyme pyroglutamyl peptidase II, which degrades the thyrotropinreleasing hormone and regulates the amount of neuropeptide reaching the anterior pituitary, as well as the enzyme thyroxine deiodinase type II, which generates bioactive triiodothyronine from thyroxine (Fekete et al. 2000; Lazcano et al. 2015). Tanycytes also seem to regulate the release of gonadotropinreleasing hormone $(\mathrm{GnRH})$ into the $\mathrm{ME}$ as their ablation prevents the pulsed release of this neuropeptide into the portal blood, the peak of luteinizing hormone and ovulation (Rodríguez et al. 2005). Despite the very important physiological roles of the tanycytes, the biology of these cells remains widely unexplored.

Cell cultures provide a simpler model to study complex biological systems that are hard to approach in vivo. The culture of hypothalamic tanycytes, however, has proved to be difficult to achieve due to several factors, including their limited amount in vivo and the cellular complexity of their normal environment, among others. Some research groups have developed methods to culture hypothalamic tanycytes (Chauvet et al. 1996; Gabrion et al. 1988). Gabrion and colleagues set up the conditions to culture dissociated cells derived from fetal mouse or rat hypothalami in serum-supplemented or chemically defined media and found that a small fraction of these cells were tanycytes. However, the major cell type present in the cultures corresponds to non-tanycytes ependymal cells, which steadily grow and form monolayers (Gabrion et al. 1988). Chauvet and colleagues also developed a culture strategy that has been extensively used by other researchers (García et al. 2003; Prevot et al. 2003). These cultures are derived from the ME of newborn 10-day-old rats and immunostaining patterns suggest that they mainly contain tanycytes (Chauvet et al. 1996). Importantly, this procedure uses medium supplemented with fetal bovine serum (FBS) and involves a secondary sub-culture. Thus, a high number of cells can be generated using this strategy but they seem to be less differentiated than in vivo tanycytes as they do not display their typical morphology. Here, we used a combination of the above referred procedures in order to obtain cell cultures enriched in tanycyte-like cells that could provide a useful tool to study this particular cell type.

\section{Materials and methods}

\section{Animals}

Sprague-Dawley rats were bred at the animal facility of the IMBICE. Animals were housed in a 12-h light/dark cycle with regular chow and water available ad libitum. This study was carried out in strict accordance to the recommendations in the Guide for the Care and Use of Laboratory Animals of the National Research Council and all efforts were made to minimize suffering. All experimentation received approval from the Institutional Animal Care and Use Committee of the IMBICE.

\section{Cell cultures}

The hypothalamic ME region was dissected from 10-day-old (P10) rats, in which tanycytes are already present lining the ventral part of the ventricle (Altman and Bayer 1978; De Seranno et al. 2004; Fig. 1a-a"). Specifically, rat brains were removed from the skull, cleaned of meningia and placed on their dorsal face to remove the median eminence-ARC region with fine-tip forceps (Fig. 1b). The optic chiasm and rostral edge of mammillary bodies were used as rostral and caudal limits, respectively, while $\sim 1 \mathrm{~mm}$ from the lateral ends of the median eminence and $1 \mathrm{~mm}$ deep were used as lateral and ventral limits, respectively (Fig. 1c, d). Then, the resulting blocks of tissue containing the ME/arcuate nucleus (ARC) region were washed three times with Hank's solution and afterwards cells were dissociated with a solution containing trypsin $0.25 \mathrm{mg} / \mathrm{ml}$ (Sigma-Aldrich, cat\# T4799) and deoxyribonuclease I (DNAse I) $0.28 \mathrm{mg} / \mathrm{ml}$ (Sigma-Aldrich, cat\# D5025,) at $37{ }^{\circ} \mathrm{C}$ for $10 \mathrm{~min}$, including a light dissociation with a glass pipette after the first $5 \mathrm{~min}$. Afterwards, the same volume of FBS (Internegocios S.A.) supplemented with DNAse I (5:1) was added to stop the digestion and the cells were spun for $5 \mathrm{~min}$ at $150 \mathrm{~g}$ and resuspended in culture medium (see composition below) plus 10\% FBS and mechanically dissociated by forcing the suspension through glass pipettes and hypodermic needles with progressively smaller diameters. After these steps, the cell number and viability was assessed in a hemocytometer by mixing the cell suspension in a 1:1 ratio with a solution of trypan blue $0.4 \%$ in saline. Cells incorporating the dye were counted as dead. The cells were then centrifuged for $5 \mathrm{~min}$ at $150 \mathrm{~g}$ and resuspended in a suitable volume of serum-free culture medium for plating. Cells were plated on either 12 -mm-diameter round cover glasses $(50,000-60,000$ cells $)$ or in $22 \times 22 \mathrm{~mm}$ cover glasses (75,000-100,000 cells). These cover glasses had been treated with poly-L-lysine $0.03 \mathrm{mg} / \mathrm{ml}$ (Sigma-Aldrich, cat\# P8920) for $1 \mathrm{~h}$ at room temperature, washed three times with sterile water and air-dried prior to use. Cells were incubated at $37^{\circ} \mathrm{C}$ in 5\% $\mathrm{CO}_{2}$ atmosphere with DMEM/F12 1:1 medium without phenol red (Microvet, cat\# P3042-001) supplemented with $0.18 \mathrm{UI} / \mathrm{ml}$ insulin (Nordisk Pharm Ind), bovine serum albumin $50 \mu \mathrm{g} / \mathrm{ml}$ (Sigma-Aldrich, cat\# A9418), transferrin $12.5 \mu \mathrm{g} / \mathrm{ml}$ (Calbiochem, cat\# 616420) and $40 \mu \mathrm{g} / \mathrm{ml}$ gentamicin sulfate salt (Richet). On day 3, half of the medium was replaced with a fresh one, plus the addition of thrombin to a 
Fig. 1 a, a' Coronal slice from a P10 rat brain that was subjected to either Nissl staining or immunofluorescence staining for vimentin-Alexa 594, respectively. A higher magnification of the region outlined in (a') is shown in (a"). Tanycytic processes are already visible and fully structured at this stage. $\mathbf{b}$ The blunt dissection of the most ventral part of the hypothalamic region (arrow) using fine-tipped forceps. Coronal slice from a P10 rat brain before (c) and after (d) the ME/ARC collection procedure. Dashed line the missing portion of tissue
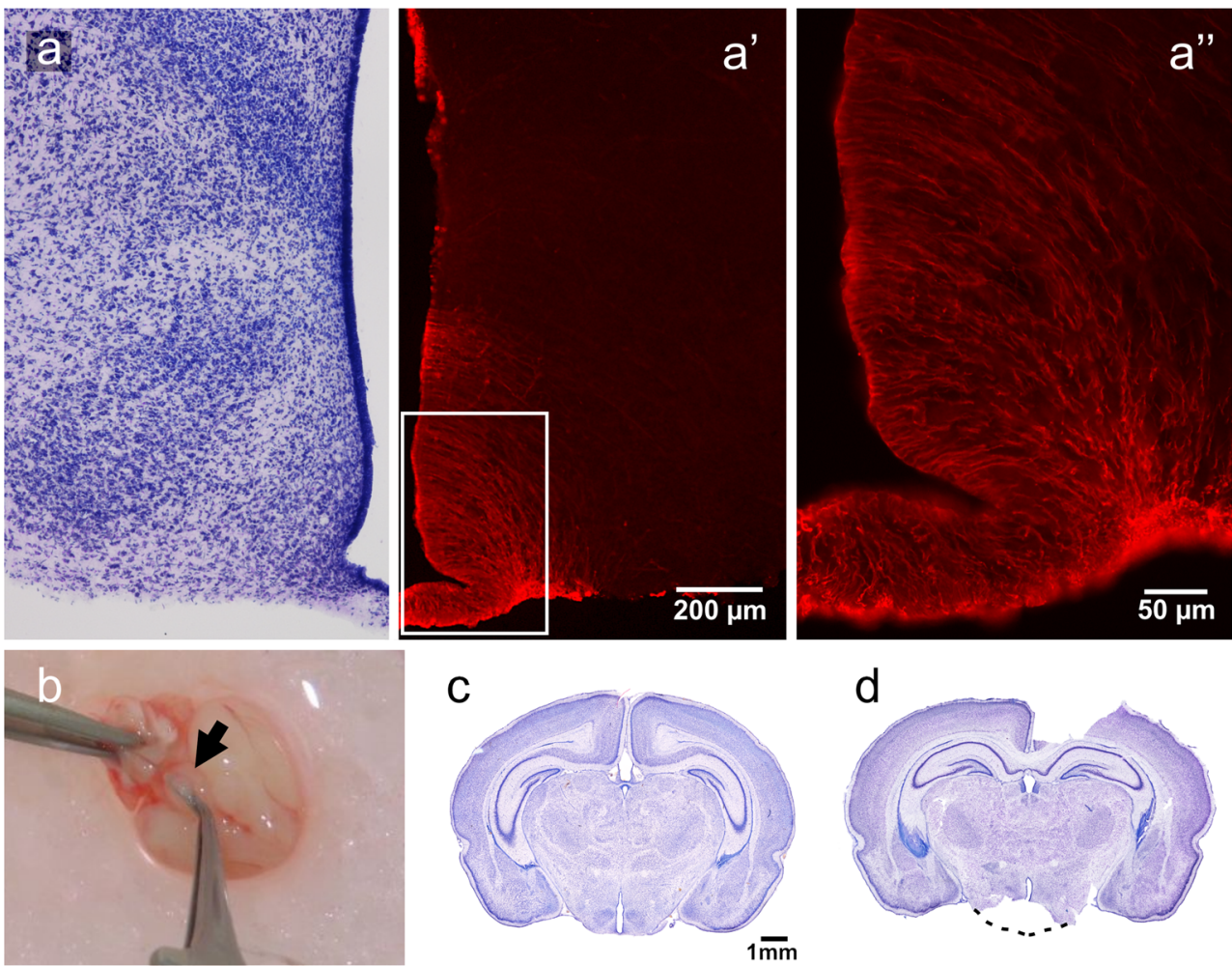

final concentration of $1.25 \mathrm{UI} / \mathrm{ml}$ (FloSeal, Baxter, cat\# 934057). Cells were cultured for a total period of 7 days.

\section{Neuroanatomical analysis of brain sections}

Intact or ME/ARC-microdissected brains were used for neuroanatomical analysis. In this case, brains were fixed overnight with $4 \%$ formaldehyde in phosphate buffer saline (PBS; pH 7.4) and then cryoprotected by overnight immersion in $20 \%$ sucrose in PBS. Coronal sections of $40 \mu \mathrm{m}$ were cut using a cryostat and used for either Nissl staining or immunohistochemistry. In the former case, the sections were mounted, stained with thionin (Sigma-Aldrich, cat\# T7029), dehydrated in an ascending alcohol series, cleared in xylene and coverslipped. For immunostaining, free floating sections were blocked with a solution containing $3 \%$ normal donkey serum and $0.25 \%$ Triton X-100 in PBS and then incubated with a chicken anti-vimentin antibody (Millipore, cat\# AB5733, 1:2000) overnight at $4{ }^{\circ} \mathrm{C}$. Then, the sections were treated with an Alexa 594 goat anti-chicken antibody (Molecular Probes, cat\# A11042, 1:2500) for $2 \mathrm{~h}$ at room temperature. Finally, the brain sections were sequentially mounted and cover-slipped with mounting medium. Fluorescence images were acquired with a Nikon Eclipse 50i microscope and a DS-Ril Nikon camera.

\section{Acridine orange staining}

Seven-day in vitro cultured cells were intravitally stained with acridine orange $1 \mu \mathrm{M}$ (Sigma-Aldrich, cat\# A6014) in culture medium for 15 min at $37{ }^{\circ} \mathrm{C}$ and washed afterwards with medium. The live-stained cells were directly observed with a Nikon TiS microscope equipped with a DS-Ri2 Nikon camera and epifluorescence and phase contrast images were taken. Acridine orange fluorescence (green to orange) was observed with a blue excitation low-pass filter cube (Nikon B-2A).

\section{Oil Red O staining}

Seven-day in vitro cultured cells were fixed with $4 \%$ paraformaldehyde in PBS at $4{ }^{\circ} \mathrm{C}$ and then washed once with PBS and once with $60 \%$ isopropyl alcohol in water. The cells were then stained in Oil Red O (Sigma-Aldrich, cat\# O0625) $3 \mathrm{mg} / \mathrm{ml}$ in $60 \%$ isopropyl alcohol for $15 \mathrm{~min}$. They were subsequently washed with $60 \%$ isopropyl alcohol, with PBS and finally cover-slipped in aqueous mountant. Cells were observed and photographed in brightfield mode with a Nikon Eclipse 50i microscope and a DS-Ri1 Nikon camera.

\section{Scanning electron microscopy analysis}

Seven-day in vitro cultured cells were fixed with $4 \%$ paraformaldehyde in PBS for $30 \mathrm{~min}$ at room temperature and then 
gradually dehydrated stepwise through ethanol from 10 to $100 \%$. The samples were dried in a critical-point $\mathrm{CO}_{2}$ drying apparatus (Baltec CP-30) and coated with gold (Jeol Fine Ion Sputter Coater JFC-1100; Jeol, Akishima). Finally, the cells were examined with a JEOL scanning electron microscope (JSM 6360 LV) and images were captured with a Nikon D90 camera.

\section{Immunocytochemical analysis}

Seven-day in vitro cultured cells were fixed with $4 \%$ paraformaldehyde in PBS for $30 \mathrm{~min}$ at $4{ }^{\circ} \mathrm{C}$. They were then treated with blocking solution (3\% normal donkey serum and $0.25 \%$ Triton X-100 in PBS) and incubated with blocking solution containing antibodies against vimentin (mouse clone V9, Biogenex AM074-10 M ready-to-use, 1/10) and either dopamine-cAMP-regulated phospho-protein (DARPP-32, goat, Santa Cruz, sc-8483, 1:50) or glial fibrillary acidic protein (GFAP, rabbit, Sigma G9269, 1:200) overnight at $4{ }^{\circ} \mathrm{C}$. Then, the cells were washed twice with PBS and incubated for $1 \mathrm{~h}$ at room temperature with the corresponding secondary antibodies conjugated to Alexa 488 or Alexa 594 (Molecular Probes, cat\# A21202 1:1000, cat\# A11058 1:500, or cat\# A21207, 1:500 in blocking solution) and rinsed with PBS. For the assessment of the presence of neuronal cells, an antibody against NeuN (Millipore, mouse Mab377, 1:400) followed by an anti-mouse Alexa 594-conjugated antibody (Molecular Pprobes cat\# A21203, 1:1000) were used. Also, for the assessment of the presence of microglial cells, an antibody against Iba1 (Wako, rabbit polyclonal cat\# 019-19741, 1:2000) followed by an Alexa 594-conjugated antibody (Molecular Probes, anti-rabbit cat\# A21207, 1:1000) were used. Cover glasses were mounted using an anti-fading solution containing bis-benzimide $10 \mathrm{mg} / \mathrm{ml}$ (Hoeschst 33258, Sigma-Aldrich, cat\# B1155) to stain the cell nuclei. The slides were visualized within a week and stored at $4{ }^{\circ} \mathrm{C}$. Fluorescence images were acquired with a Nikon Eclipse 50i microscope and a DS-Ril Nikon camera. FIJI was used to adjust the contrast and brightness of the microphotographs and to prepare the composite panels.

\section{Electrophysiological analysis}

Tanycyte-like cells were patched in a whole-cell configuration between 5 and 12 days in culture. The external solution composition was (in $\mathrm{mM}$ ): $135 \mathrm{NaCl}, 4.7 \mathrm{KCl}, 1.2 \mathrm{MgCl}_{2}, 2.5$ $\mathrm{CaCl}_{2}, 10$ HEPES and 10 glucose, $\mathrm{pH}=7.4$. The internal solution composition was (in $\mathrm{mM}$ ): 140 potassium gluconate, 5 $\mathrm{KCl}_{2}, 5 \mathrm{NaCl}, 10$ HEPES, $4 \mathrm{MgCl}_{2}$, 3 Na-ATP, 0.3 Na-GTP and 0.6 EGTA, $\mathrm{pH}=7.2$. Borosilicate glass capillaries were used to generate the micropipettes. Leak currents less than 50 $\mathrm{pA}$ and resistances between 3 and $6 \mathrm{M} \Omega$ when electrodes were filled with internal solution were accepted. An Axopatch 200 amplifier (Molecular Devices) was used and data were sampled at $20 \mathrm{kHz}$ and filtered at $10 \mathrm{kHz}(-3 \mathrm{~dB})$ using PCLAMP8.2 software. Measurement of resting membrane potential was done in track mode and membrane potential values that remained constant for at least 5 min during the recordings were included. These measurements were not corrected for the liquid junction potential. Voltage change recordings were done in current clamp mode using current injections from -100 to $+100 \mathrm{pA}$ in square pulses of $200 \mathrm{~ms}$.

\section{Endocytic activity analysis}

Seven-day in vitro cell cultures were exposed to a suspension of $2 \mu \mathrm{g} / \mathrm{ml}$ carboxylate-modified 40-nm red fluorescent microspheres (Fluospheres, Molecular Probes, cat\# F8793) in culture medium at $37^{\circ} \mathrm{C}$ or $4{ }^{\circ} \mathrm{C}$. After $15 \mathrm{~min}$, the cells were washed twice with PBS, fixed with $4 \%$ paraformaldehyde in PBS for 30 min at $4{ }^{\circ} \mathrm{C}$ and mounted with anti-fading solution containing bis-benzimide $10 \mathrm{mg} / \mathrm{ml}$. Widefield fluorescence image $Z$-stacks were acquired in 200 -nm steps with a motorized automated rig on a Nikon Eclipse 50i microscope using a DS-Ril Nikon camera. The widefield datasets were deconvolved with Huygens (SVI). Immunostaining for Rab5 was also performed for the $37{ }^{\circ} \mathrm{C}$ condition following a procedure similar to that described above. A rabbit antibody against Rab5 (Cell Signaling, cat \#3547, 1:1000) and an anti-rabbit Alexa 488-conjugated antibody (Invitrogen, cat\# A-11008, 1:1000) were used. Images were acquired using a Zeiss AxioObserver D1 with an Apotome. 2 module. FIJI was used to generate maximum intensity and orthogonal projections.

\section{Statistical analysis}

Twelve independent sets of cultures, which included a total of 135 pups, were performed using the above-described conditions. The analysis of each type of imaging study was performed on a minimum of 20-40 cells per cover glass, using 4-6 cover glasses that were derived from at least two different experiments. Data are expressed as the mean \pm SEM.

\section{Results}

\section{Overall performance of primary cultures}

Typical average yield of the tissue dissociation was estimated in 60,000-90,000 cells per ME/ARC region collected. Cell viability monitored by trypan blue indicated that the vast majority of the cells were alive right before seeding. Cell cultures were plated using a density of $\sim 150$ cells $/ \mathrm{mm}^{2}$ (i.e., 75,000 cells in a $22 \times 22 \mathrm{~mm}$ cover glass). A few minutes after plating, most cells were attached and showed a spherical 
morphology with some of them already displaying a single and short cellular process. After 1 day in vitro, tanycyte-like and non-tanycyte-like cells were observed surrounded by aggregates of cell debris and dead cells. After 3 days in vitro, most cell debris and dead cells were removed when replacing part of the medium by fresh culture medium containing thrombin. After 4 days in vitro, a density of $\sim 25$ cells $/ \mathrm{mm}^{2}$ remained alive and healthy in the culture as indicated by phase contrast microscopy. Neither the cell density nor the overall appearance of the cell cultures changed significantly over the next 5 days in vitro and, therefore, the characterization of the cultured cells was performed by day 7 in vitro.

\section{Morphological characterization of tanycyte-like cells}

At day 7 in vitro, a cell population with tanycyte-like morphology could be easily visualized by phase contrast microscopy analysis (Fig. 2a-a'). The tanycyte-like cells represented around $20-30 \%$ of the total number of cells. The nontanycytes-like cells included a heterogeneous group of flat and polygonal epithelial-like cells, round epithelial-like cells, fibroblast-like cells and macrophage-like cells. Importantly, no ciliated cells were found in the cell cultures, as assessed by high-magnification phase contrast observation. In addition, neither NeuN- nor Iba1-immunoreactive cells were found in the cultures, suggesting that neuronal and microglial cells were not present in the cultures. Tanycyte-like cells represented a highly homogenous set of cells that displayed a nonciliated cell body and a long cytoplasmic shaft. The cell body of the tanycyte-like cells was slightly elongated (average length $41.5 \pm 1.8 \mu \mathrm{m}$, average width $19.5 \pm 0.9 \mu \mathrm{m}$ ) and displayed an eccentric nucleus. The process of the tanycytelike cells varied both in length and diameter and typically ended as a swollen varicosity or footplate, which was never found in association with other cells of the culture. The cell length displays an asymmetric unimodal distribution with a median value of $124 \mu \mathrm{m}$ (25\% percentile: $93 \mu \mathrm{m}$; $75 \%$ percentile: $190 \mu \mathrm{m}$ ) and a small fraction of cells extending past the 500- $\mu \mathrm{m}$ range. Phase contrast analysis showed that tanycyte-like cells contained abundant highly refractive intracellular vesicle-like structures, which were bigger in the cell body and smaller in the processes (see Fig. 2a'). Within the cell body, these structures were preferentially located at the pole where the shaft forms as well as at the apical end. In order to test if these vesicles were acidic, such as lysosomes or late endosomes, cells were processed for acridine orange staining, which showed orange fluorescent compartments within the cell body, in most cases surrounding the nucleus, as well as in some small sections along the cell shaft and in the terminal foot plate (Fig. 2b-b"'"). Notably, none of the highly refractive vesicles were of acidic nature upon comparison of fluorescence with phase contrast images. Since tanycytes are known to contain lipids droplets (Flament-Durand and Brion
1985; Peruzzo et al. 2004; Rodríguez et al. 2005), the cells were also stained with the fat-soluble dye Oil Red O (Fig. 2c-c"). Oil red O staining revealed a strong red staining pattern coincident with the distribution of these highly refractive structures, which indicates the lipid-containing nature of these vesicles.

Scanning electron microscopy analysis showed that the cell body of tanycyte-like cells displayed small spherical protrusions (or blebs) and extensive adhesion processes to the glass surface, while their cytoplasmic shafts displayed thin adhesion processes with small branching extensions, especially at the most distal part (Fig. 2d-e"). Thus, the experimental conditions described in the current study allow obtaining a cell culture containing tanycyte-like cells that resemble some morphological features of tanycytes in vivo.

\section{Immunocytochemical characterization of tanycyte-like cells}

Cell cultures were characterized by immunocytochemical methods in order to test the presence of cell markers known to be present in tanycytes in vivo. All tanycyte-like cells were markedly positive for vimentin, even though this staining was also present in most of the other cells within the culture. This finding should be interpreted with caution, as vimentin can be induced in astroglial primary cultures by in vitro culture environments (Ciesielski-Treska et al. 1988). All tanycyte-like cells were also immunoreactive for GFAP; however, the intensity of this signal was weaker as compared to the signal observed in a small fraction of cells present in culture that did not possess a tanycyte-like morphology (Fig. 3a-c"). As expected, vimentin and GFAP stainings displayed a fairly similar pattern since both proteins have the same subcelular localization as part of the type III intermediate filaments and are known to form heteropolymers (Abd-el-Basset et al. 1992). Importantly, 90\% of tanycytelike cells positive for vimentin were also positive for DARPP-32 (Fig. 3d-d"), while the rest of the cells were not immunoreactive for this marker.

\section{Electrophysiological characterization of tanycyte-like cells}

Whole-cell patch-clamp recordings of cultured tanycyte-like cells indicated that they display an average resting membrane potential of $-49.9 \pm 4.7 \mathrm{mV}(n=13$;;Fig. $4 \mathrm{a}, \mathrm{b})$. As expected, tanycyte-like cells failed to display either spontaneous fluctuations in their membrane potential or spike-like voltage changes in response to current injections ranging from -100 to $+100 \mathrm{pA}$ (Fig. 4c). Thus, tanycyte-like cells display the electrophysiological response expected for this subtype of glial cell. 


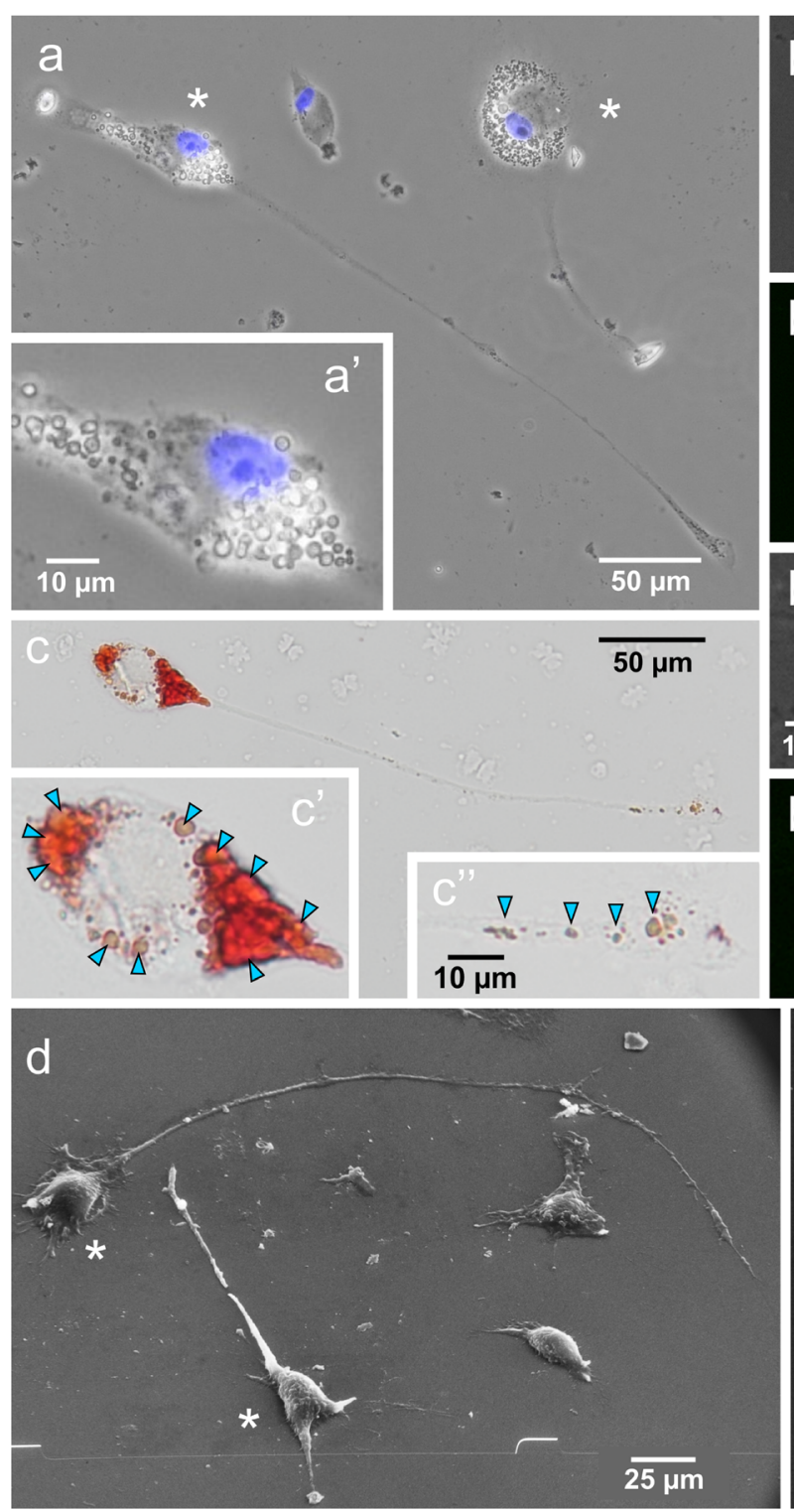

Fig. 2 Morphological and ultrastructural characterization of tanycytelike cells. a Representative phase contrast microscopy image from cells present in the cultures from day 7. The grayscale image is superimposed with a Hoechst fluorescent nuclear stain (blue). Asterisks label tanycytelike cells, while the other cell present in the image is a non-tanycyte-like cell. A magnified inset is presented in (a'), showing the body of one of these cells where the intracellular presence of highly refractive vesiclelike structures is evident. b Representative fluorescence image of a tanycyte-like cell after acridine orange staining. A corresponding phase contrast ( $\mathbf{b}^{\prime}$ ) image is shown, along with paired insets depicting the cell body (b", b"') and the terminal portion of the projection with its foot plate (b"', b"'"). Cyan arrowheads point at highly refractive vesicle-like structures, while magenta arrows point at acidic acridine orange-loaded
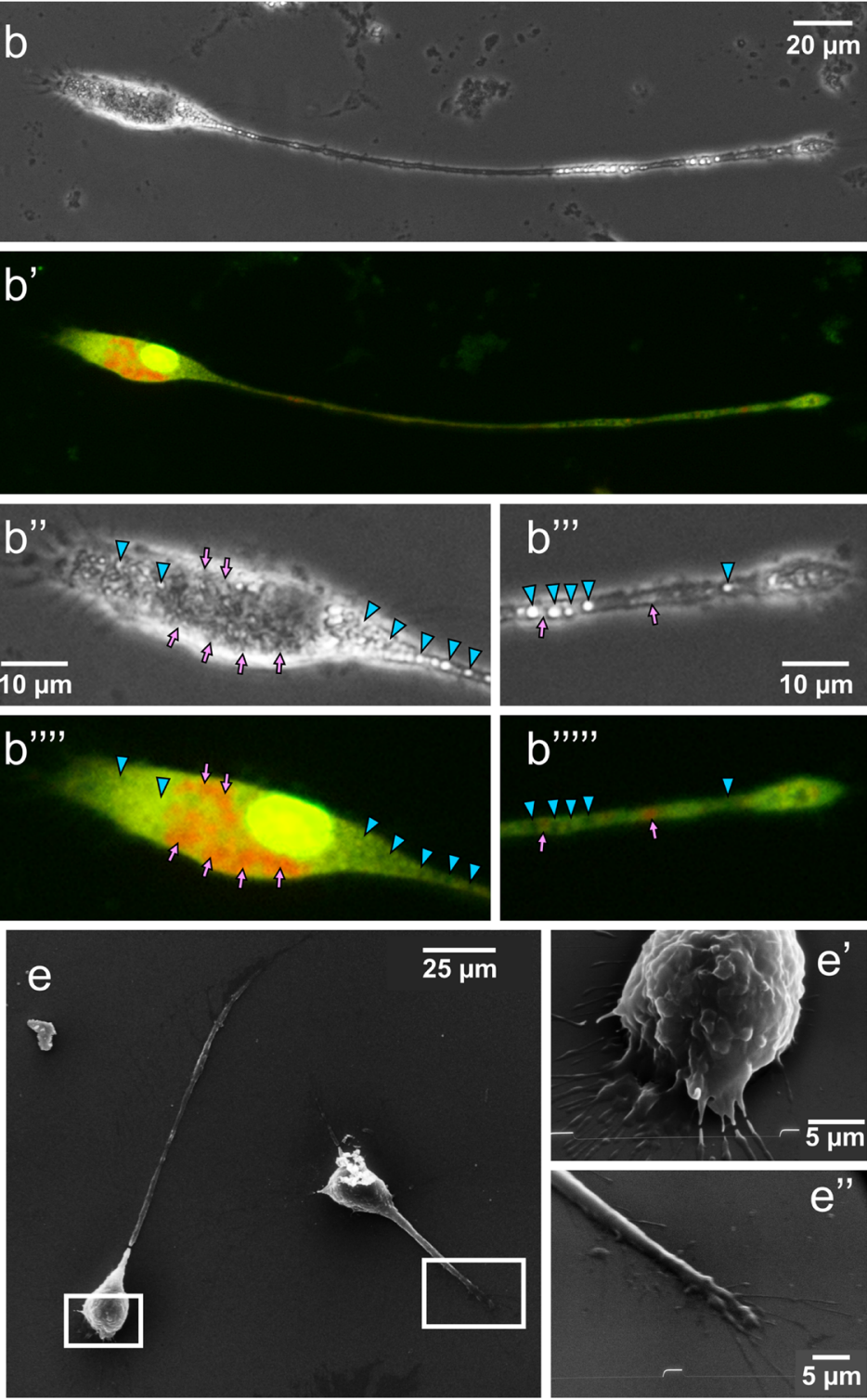

structures. c Representative image of a tanycyte-like cell after staining with Oil Red O. Insets show a magnified view of the cell body ( $\left.\mathbf{c}^{\prime}\right)$ and the terminal portion of the shaft (c"). Cyan arrowheads point at Oil Red Ostained vesicle-like structures. d Representative low-magnification scanning electron microscopy image from cells present in the cultures from day 7. Tanycyte-like cells are labeled with an asterisk and present small branching extensions all along their body length.e Long and middlelength tanycyte-like cells, along with higher magnification details (e'e"). For the former, very thin adhesion processes are clearly visible at the distal end. The detail of the cell body shown in (e') presents small cytoplasmic blebs and extensive adhesion processes. Magnified in (e"), the most distal part of the shaft has clear varicosities and branching

modified red fluorescent microspheres led to the appearance of a punctate signal pattern within the cell bodies of several cell types within the culture including the tanycytelike cells (Fig. 5d-f). This intracellular punctate fluorescent pattern was not observed when primary cultures were incubated with fluorescent microspheres at $4{ }^{\circ} \mathrm{C}$ (Fig. 5a-c), 

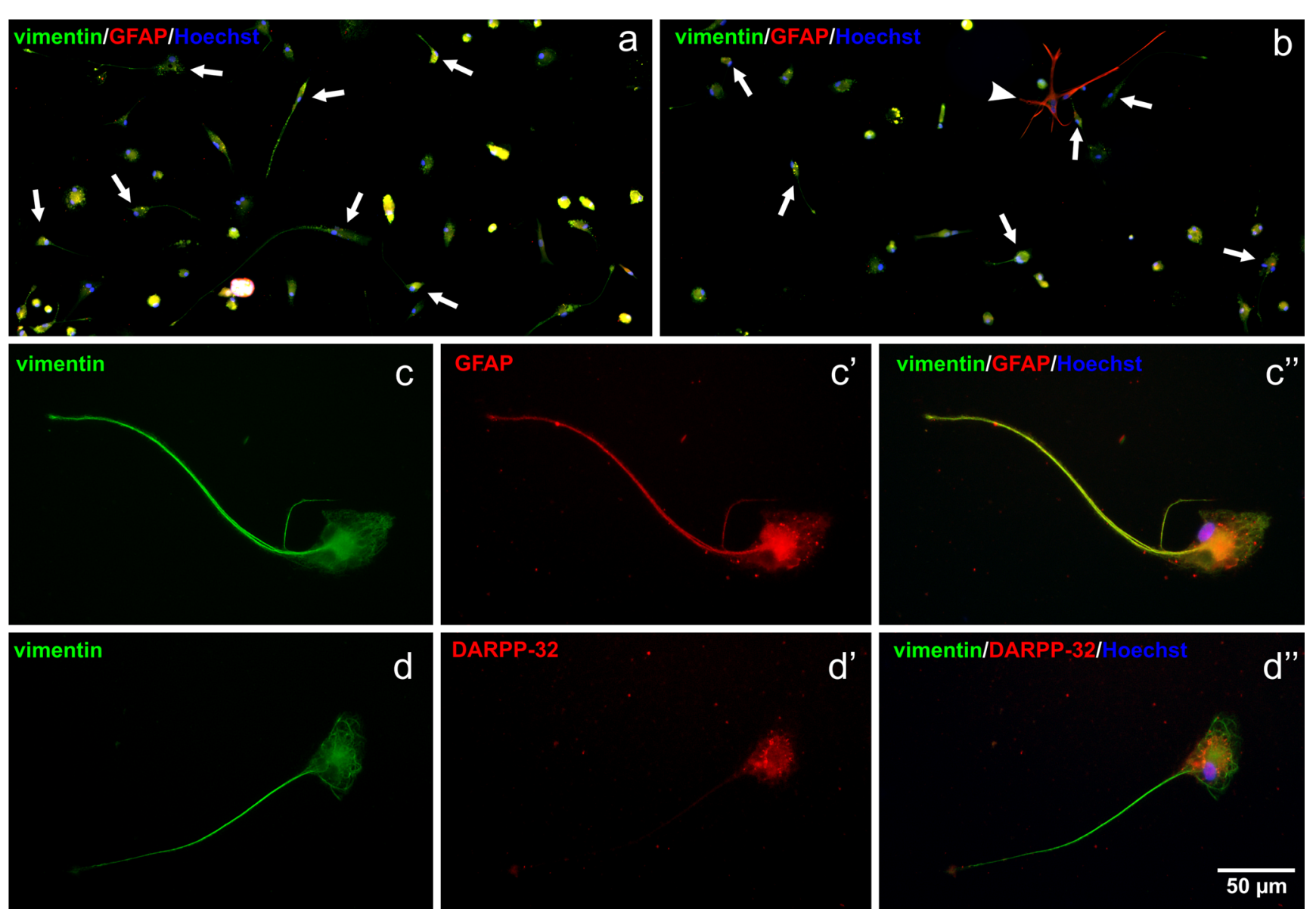

Fig. 3 Immunocytochemical characterization of tanycyte-like cells. a, b Low-magnification images of cultures co-stained for vimentin and GFAP. Several tanycyte-like cells can be observed, indicated by arrows. A group of strongly GFAP-positive non-tanycytes are indicated in (b) with an arrowhead. c-c" Representative image of a typical tanycyte-like cell

where a double immunofluorescent staining for vimentin and GFAP was performed. d-d" A typical tanycyte-like cell as seen after doubleimmunofluorescent staining for vimentin and DARPP-32. Composite images of the double staining plus nuclear Hoechst signal are also provided for each case

a temperature known to block active vesicular trafficking pathways. In order to test if internalized molecules were located in an endocytic compartment, we performed immunostaining against Rab5 in cell cultures previously incubated with microspheres at $37{ }^{\circ} \mathrm{C}$. We found that a

significant fraction of the intracellular red fluorescent punctate co-stained with this early endocytic marker (Fig. 5g-j). Thus, tanycyte-like cells seem to display active endocytic processes under the experimental conditions described here.
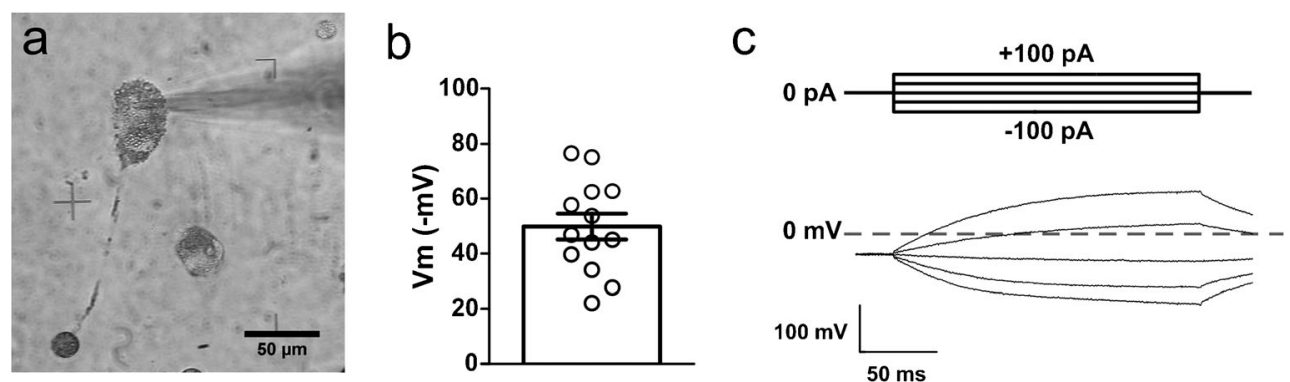

Fig. 4 Electrophysiological characterization of tanycyte-like cells. a Representative image of a typical tanycyte-like cell accessed by the pipette during recording. b Distribution of membrane potential values observed in cultured tanycyte-like cells. c Representative voltage traces in

response to current injection performed on these cells. Recordings were done in current clamp mode applying current injections from -100 to $+100 \mathrm{pA}$ in square pulses of $200 \mathrm{~ms}$ 

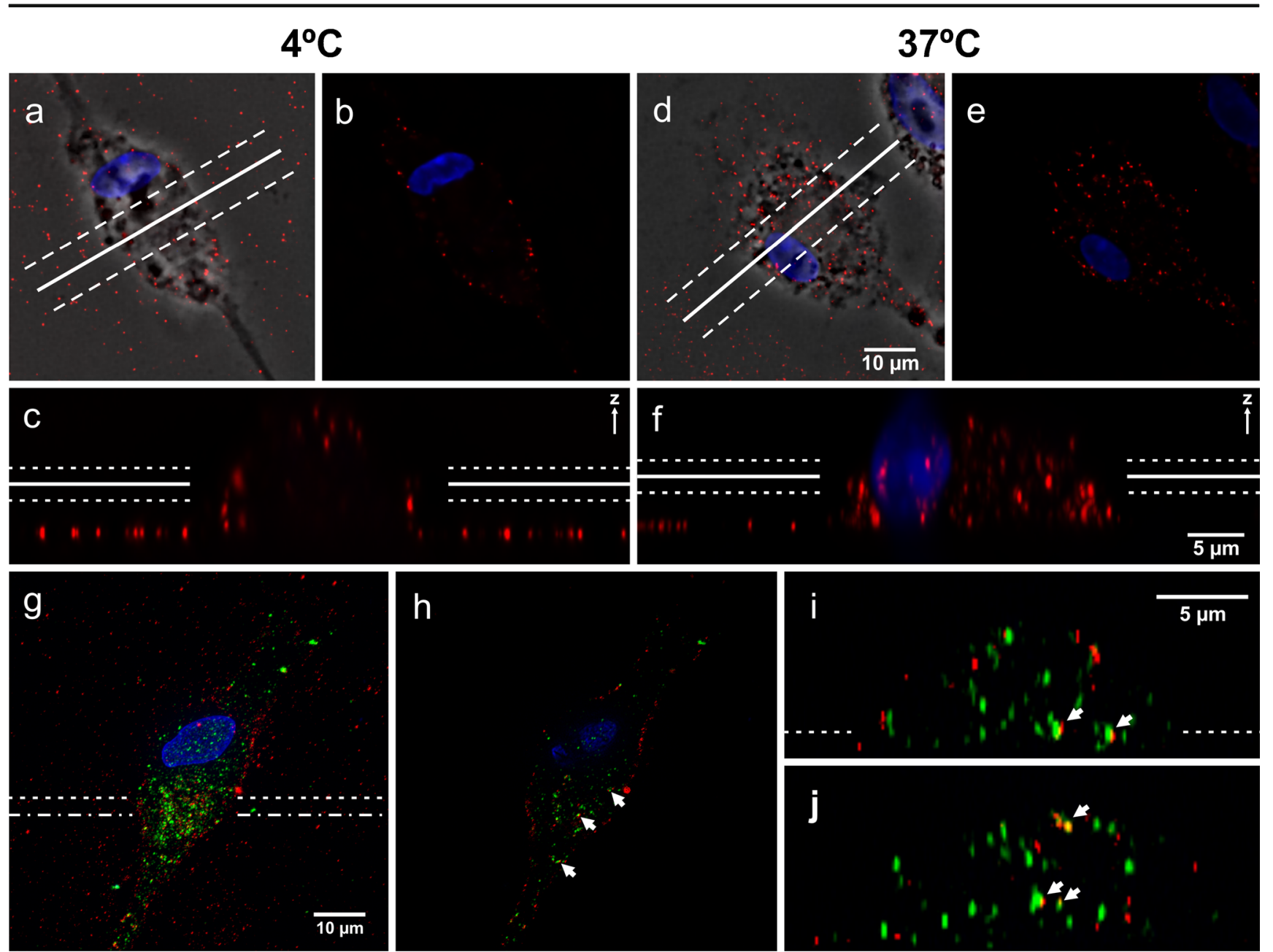

Fig. 5 Endocytic activity of tanycyte-like cells. Cultured tanycyte-like cells were incubated in the presence of red fluorescent microspheres for 15 min at $4{ }^{\circ} \mathrm{C}$ or $37^{\circ} \mathrm{C}$, fixed and a fluorescence image stack was acquired at 200-nm intervals and deconvolved. a, d Maximum intensity projection of the stacks for the $4{ }^{\circ} \mathrm{C}$ and $37{ }^{\circ} \mathrm{C}$ conditions, respectively. Corresponding phase contrast and Hoechst nuclear stain images are superimposed to better delineate the cell contour. b, e Maximum intensity projection of the same stacks but only including a $2-\mu \mathrm{m}$ range starting $2 \mu \mathrm{m}$ away from the glass slide, so to only include the bulk of the cytoplasm of each cell. The cell incubated at $4{ }^{\circ} \mathrm{C}$ only displays a punctate red fluorescent signal at the periphery, while the cell incubated at $37{ }^{\circ} \mathrm{C}$ also shows abundant intracellular signal. For these same cells, $\mathbf{c}, \mathbf{f}$ show a $10-$

\section{Discussion}

Here, we used a combination of rigorous sample collection, based on neuroanatomical criteria as well as on the age of the animals, together with specific and defined culture conditions, in order to culture cells that resemble in vivo tanycytes in terms of structural features, molecular markers and biological activity. Thus, we provide a simple and reproducible method for the primary culture of rat hypothalamic tanycyte-like cells. To our knowledge, this is the first in vitro system in which tanycyte-like cells can be individually identified and used to investigate some aspects of the cell biology of tanycytes that

$\mu \mathrm{m}$-wide orthogonal maximum intensity projection of planes passing through the middle portion of the cell body. Again, only the cell incubated at $37{ }^{\circ} \mathrm{C}$ show an extensive red fluorescent signal that corresponds to intracellular locations. Corresponding center slice and projection range for (b) and (e) are indicated in (c) and (f) with continuous and dashed lines, while the same is done for $(\mathbf{c})$ and $(\mathbf{f}),(\mathbf{a})$ and $(\mathbf{b})$. $\mathbf{g}-\mathbf{j}$ show a similar experiment performed at $37^{\circ} \mathrm{C}$ with fluospheres (red) in which an antiRab5 staining (green) was performed afterwards. g Maximum intensity projection. $\mathbf{h}$ Single image form the stack, at the level indicated by dashed line in (i). $\mathbf{i}, \mathbf{j}$ Orthogonal views at the levels indicated in (g) with dashed and dot-dashed lines, respectively. Arrows indicate fluospheres associated with Rab5 labeling

require morphologically conserved cells, such as the mechanisms mediating endocytosis or transcytosis.

The age of the animals at which we obtained the tissue for cultures was based on previous studies (Chauvet et al. 1996; Prevot et al. 2003) and seemed to be a critical aspect of the procedure. Thus, despite tanycyte cultures having been described from earlier stages (Gabrion et al. 1988, 1998; Jordan et al. 1990), the P10 stage has resulted in the most successful and reproducible cultures in our hands. Here, we confirmed that the anatomical organization and the immunostaining patterns of hypothalamic tanycytes of 10-day-old pups were very similar to those seen in adult rats. Similarly 
to other central nervous system cells, the aptitude of tanycytelike cells to be cultured in vitro rapidly decreases with the age of the donor tissue (Chauvet et al. 1996). Indeed, we were unable to culture tanycyte-like cells from adult rodents (unpublished observations). The reason why dispersed tanycytelike cells obtained from adult brains poorly survive the culture procedure is unclear but presumably involves the fact that mature tanycytes are more susceptible to damage during dissociation, as has been already noted for glial and neuronal cell types (Chauvet et al. 1996). Although tanycytes could also be cultured from ME/ARC tissue obtained from late embryonic stages, these cell cultures are less enriched in tanycyte-like cells, likely due to a combination of facts including that the neuroanatomical limits to dissect the ME/ARC are harder to distinguish at prenatal stages and that the mitotic potential of other glial cells is higher at these ages (Rice and Barone 2000). In addition, tanycytes from embryonic brains may not behave as mature tanycytes since the ME functionality fully develops post-natally (Peruzzo et al. 2000). In balance, ME/ARC tissue from 10-day-old pups provides an excellent source of tanycytes as they remain plastic enough to resist dissociation as well as further in vitro survival and also they better resemble mature tanycyte physiology.

Here, we used a defined medium for culturing hypothalamic tanycytes, a strategy that is in clear contrast to most of the previously described protocols that used culture media supplemented with animal serum. Chemically defined media allow the reduction of variability in the procedure, since all components of the system are specifically known. The defined medium was composed of DMEM:F12 supplemented with serum albumin, insulin, transferrin and gentamycin. As reported previously (Gabrion et al. 1998), thrombin was added to the culture medium from day 3 . Thrombin is a serine protease involved in the coagulation cascade that improves the viability of ependymal cells without any effect on oligodendrocytes, macrophages and fibroblasts proliferation and has been described to act through the activation of the protease-activated receptor 1 (Perraud et al. 1987; Tritschler et al. 2007). Transferrin, serum albumin and insulin are common supplements to serum-free media and have been widely used in ependymal serum-free cultures (Gabrion et al. 1998; Grondona et al. 2013; Laabich et al. 1989; Tritschler et al. 2007; Weibel et al. 1984, 1986). Notably, culture conditions seem to mainly favor the survival of tanycyte-like cells, since neurons, microglia and ciliated ependymal cells were not present in the cell cultures. However, culture conditions also seem to abrogate both the proliferative capacity of tanycytes, since the number of tanycyte-like cells was not increased during the cultures (see Chiasson et al. 1999) and their progenitor capabilities and neurogenic potential (Robins et al. 2013), since no neurospheres developed. We hypothesize that the lack of key growth factors in the serum-free medium blunted the proliferative potential of any cell type present in the culture and might be responsible for the relatively low surface density of cells achieved. An intriguing possibility is that this low surface density is what fosters the differentiation of single tanycyte-like cells as seen in our culture conditions. Thus, the current method seems to be a reproducible strategy to culture isolated tanycytelike cells that were already present in the in vivo context. Notably, $\sim 70-80 \%$ of the cultured cells did not display a tanycyte-like morphology. These non-tanycyte-like cells, which scarcely divide or interact with other cells, likely represented a heterogeneous population of quiescent glial cells, which may include other subtypes of ependymal cells. The presence of other cells, different from tanycytes, is inherent to the culture system and restricts its use to methodological approaches that involve assessments in individual cells, such as imaging techniques or electrophysiological recordings.

At the electrophysiological level, we found that tanycytelike cells showed a steady resting membrane potential and lacked spontaneous or evoked electrical excitability. We obtained a mean value of $-50 \mathrm{mV}$, which is similar to the one previously informed for cultured tanycytes. Hild and co-workers were the first to measure resting membrane voltage in cultured rat ependymal cells, reporting values between -40 and $-60 \mathrm{mV}$ (Hild et al. 1965). Later on, Jarvis and Andrew reported that rat tanycytes from brain slices displayed a membrane potential $\sim-80 \mathrm{mV}$ and presumed that the less negative values obtained in cultured tanycytes were due to membrane damage (Jarvis and Andrew 1988). Based on our recording conditions with minimum leak current and series resistance, we suggest that the difference of the membrane potential of tanycytes recorded in brain slices and in culture is related to intrinsic aspects of each experimental model. One plausible explanation is that gap junction complexes, which closely connect tanycytes in vivo, function as hemichannels in culture conditions and partially contribute to depolarize them, since these hemichannels can display non-selective cationic currents under some conditions (Bennett et al. 2003). Moreover, we found no electrical spike-like events in tanycyte-like cells, whether spontaneous or in response to an intracellular injection of current. Thus, the electrical properties of in vitro tanycyte-like cells agree with previous observations performed in this type of glial cell both in vitro and ex vivo and are clearly distinct from the ones expected for a neuronal phenotype.

Tanycytes do not constitute a homogeneous cell population in the rat, in which four types of them have been distinguished (Rodríguez et al. 2005). Such morphological diversity of hypothalamic tanycytes in vivo likely reflects differences in their functions. Thus, tanycytes are classified as $\alpha 1, \alpha 2, \beta 1$ or $\beta 2$ depending on their location, morphology, molecular markers, ultrastructure and functions. The $\alpha 1$ tanycytes are located in the middle portion of the ventricle and project their process to the ventromedial nucleus, while the $\alpha 2$ tanycytes are observed ventrally and project into the ARC. The $\beta 1$ and $\beta 2$ tanycytes are, respectively, located at the lateral or medial portion of the 
floor of the third ventricle and send their projections ventrolaterally $(\beta 1)$ or ventrally $(\beta 2)$ to the plexus of portal fenestrated capillaries located in the external layer of the ME. The tanycyte-like cells cultured in the described conditions display several features that resemble those of in vivo tanycytes. At a morphological level, tanycyte-like cells displayed a medium size cell body and a single and long process that ends in a footplate that bears an overall resemblance to that seen in vivo, although lacking the extensive branching usually observed. In terms of the molecular markers for tanycytes, cultured tanycyte-like cells displayed vimentin and DARPP-32 immunoreactive signals, as well as weak GFAP immunoreactivity. Vimentin labeling is a common feature to all ependymal cells, including tanycytes (Chauvet et al. 1998). GFAP expression in tanycytes has been previously demonstrated (Bascó et al. 1981; de Vitry et al. 1981); however, the immunoreactive signal for GFAP in tanycytes is much lower than in astrocytes (Chauvet et al. 1995, 1996; Meister et al. 1988) and, consequently, tanycytes have sometimes been regarded as negative for this marker (Chauvet et al. 1998; de Vries et al. 2014). More recently, GFAP expression in mice tanycyte subtypes has been mapped and mainly found in dorsal $\alpha 2$ and some $\alpha 1$ subtypes, while little or no signal was seen in ventral $\alpha 2$ or $\beta$ subtypes (Robins et al. 2013). Thus, our finding of weak GFAP staining in cultured tanycyte-like cells together with their strong vimentin immunoreactivity is in agreement with their origin from the mediobasal hypothalamus and suggests that they are more ventral tanycyte subtypes, namely $\alpha 2$ or $\beta$. Importantly, DARPP-32 is only expressed in the hypothalamic tanycytes of the adult rat brain (Meister et al. 1988; Ouimet et al. 1984). Thus, the presence of DARPP-32 in cultured tanycyte-like cells strongly indicates that they are indeed tanycytes.

Notably, in vitro tanycyte-like cells contained numerous and large lipid inclusions. Interestingly, in vivo, only $\beta 1$ tanycytes contained large lipid droplets, which are in close association with the smooth endoplasmic reticulum (Rodríguez et al. 1979). These inclusions are thought to be involved in the synthesis of prostaglandins, which in turn regulates GnRH release in the ME (Rodríguez et al. 2005). Although it is unclear if the presence of the lipid droplets in all tanycyte-like cells is due to the culture system or if the culture conditions favor the survival of some subtype/s of tanycytes, the fact that our tissue collection procedure mainly involves the medio-basal hypothalamus would agree with the idea that the presently reported cultures are enriched in $\beta$ tanycytes.

Scanning electron microscopy analysis allowed the observation of key morphological features of in vitro tanycyte-like cells that can be compared with previous characterizations of tanycytes in situ. Thus, the cell body of the in vitro tanycytelike cells displayed prominent blebs or spherical protrusions, which were described in the apical surface of tanycytes in vivo, by both scanning and transmission electron microscopy (Jarvis and Andrew 1988; Paull et al. 1977; Rinne 1966; Rodríguez et al. 1979; Wittkowski 1998). These protrusions facing the third ventricle had been prominently observed in ventral tanycytes, namely $\beta$, which further supports the notion that our cultures are enriched in this tanycyte subtype. Membrane blebbing is not only seen in in vivo tanycytes but also in any cell type during apoptosis. Since in vitro tanycyte-like cells fail to show any other morphological feature associated with apoptosis (e.g., cell shrinkage or nuclear fragmentation), the presence of blebs seems to be more suggestive of endocytic activity (Rodríguez et al. 2005). The presence of numerous acidic vesicles, which are presumably lysosomes or late endosomes, in the cell body of in vitro tanycyte-like cells is also consistent with the existence of an endocytic machinery. In line with these morphological features, tanycyte-like cells displayed uptake of extracellular material when the cell cultures were exposed to fluorescent beads. Furthermore, the presence of immunostaining against Rab5 associated with the intracellularly incorporated beads strongly suggests that this uptake indeed took place by endocytosis. Both clathrin- and caveolin-mediated endocytosis have been reported as the main mechanisms for the active uptake of small-sized $(20-50 \mathrm{~nm})$ polystyrene beads in vitro, depending on cell lineage (dos Santos et al. 2011; Rejman et al. 2004; Smith et al. 2012). Notably, these two endocytic mechanisms are not considered to be equally present in all tanycyte subtypes (Peruzzo et al. 2004; Rodríguez et al. 2005). Thus, the described in vitro system could be valuable to gain insight into the molecular mechanisms mediating the endocytic and transcytotic properties of these cells. Future studies using specific markers for each tanycyte subtype (see Goodman and Hajihosseini 2015) as well as functional evaluations may help to better characterize the nature of the cells reported here.

Acknowledgements This work was supported by grants of the National Agency of Scientific and Technological Promotion of Argentina to MP (PICT2011-2142) and to JR (PICT2013-1145).

We would like to thank Dr. Maria José Bellini, Lic. Micaela García and Dr. Camila Scorticati for kindly providing some of the antibodies for this work.

\section{Compliance with ethical standards}

Conflict of interest The authors declare that they have no conflict of interest.

\section{References}

Abd-el-Basset EM, Ahmed I, Kalnins VI, Fedoroff S (1992) Immunoelectron microscopical localization of vimentin and glial fibrillary acidic protein in mouse astrocytes and their precursor cells in culture. Glia 6:149-153. doi:10.1002/glia.440060209 
Altman J, Bayer SA (1978) Development of the diencephalon in the rat. III. Ontogeny of the specialized ventricular linings of the hypothalamic third ventricle. J Comp Neurol 182:995-1015

Balland E, Dam J, Langlet F, Caron E, Steculorum S, Messina A, Rasika S, Falluel-Morel A, Anouar Y, Dehouck B, Trinquet E, Jockers R, Bouret SG, Prévot V (2014) Hypothalamic tanycytes are an ERKgated conduit for leptin into the brain. Cell Metab 19:293-301. doi: 10.1016/j.cmet.2013.12.015

Bascó E, Woodhams PL, Hajós F, Balázs R (1981) Immunocytochemical demonstration of glial fibrillary acidic protein in mouse tanycytes. Anat Embryol (Berl) 162:217-222

Bennett MVL, Contreras JE, Bukauskas FF, Sáez JC (2003) New roles for astrocytes: gap junction hemichannels have something to communicate. Trends Neurosci 26:610-617. doi:10.1016/j.tins.2003.09. 008

Chauvet N, Parmentier ML, Alonso G (1995) Transected axons of adult hypothalamo-neurohypophysial neurons regenerate along tanycytic processes. J Neurosci Res 41:129-144. doi:10.1002/jnr.490410115

Chauvet N, Privat A, Alonso G (1996) Aged median eminence glial cell cultures promote survival and neurite outgrowth of cocultured neurons. Glia 18:211-223. doi:10.1002/(SICI)1098-1136(199611)18: $3<211::$ AID-GLIA5 $>3.0 . \mathrm{CO} ; 2-1$

Chauvet N, Prieto M, Alonso G (1998) Tanycytes present in the adult rat mediobasal hypothalamus support the regeneration of monoaminergic axons. Exp Neurol 151:1-13. doi:10.1006/exnr.1998.6784

Chiasson BJ, Tropepe V, Morshead CM, van der Kooy D (1999) Adult mammalian forebrain ependymal and subependymal cells demonstrate proliferative potential, but only subependymal cells have neural stem cell characteristics. J Neurosci 19:4462-4471

Ciesielski-Treska J, Goetschy JF, Ulrich G, Aunis D (1988) Acquisition of vimentin in astrocytes cultured from postnatal rat brain. J Neurocytol 17:79-86

De Seranno S, Estrella C, Loyens A, Cornea A, Ojeda SR, Beauvillain JC, Prevot V (2004) Vascular endothelial cells promote acute plasticity in ependymoglial cells of the neuroendocrine brain. J Neurosci 24:10353-10363. doi:10.1523/JNEUROSCI.3228-04.2004

de Vitry F, Picart R, Jacque C, Tixier-Vidal A (1981) Glial fibrillary acidic protein. A cellular marker of tanycytes in the mouse hypothalamus. Dev Neurosci 4:457-460

de Vries EM, Kwakkel J, Eggels L, Kalsbeek A, Barrett P, Fliers E, Boelen A (2014) NFKB signaling is essential for the lipopolysaccharide-induced increase of type 2 deiodinase in tanycytes. Endocrinology 155:2000-2008. doi:10.1210/en.20132018

dos Santos T, Varela J, Lynch I, Salvati A, Dawson KA (2011) Effects of transport inhibitors on the cellular uptake of carboxylated polystyrene nanoparticles in different cell lines. PLoS ONE 6, e24438. doi: 10.1371/journal.pone.0024438

Fekete C, Mihály E, Herscovici S, Salas J, Tu H, Larsen PR, Lechan RM (2000) DARPP-32 and CREB are present in type 2 iodothyronine deiodinase-producing tanycytes: implications for the regulation of type 2 deiodinase activity. Brain Res 862:154-161

Flament-Durand J, Brion JP (1985) Tanycytes: morphology and functions: a review. Int Rev Cytol 96:121-155

Gabrion J, Peraldi S, Faivre-Bauman A, Klotz C, Ghandour MS, Paulin D, Assenmacher I, Tixier-Vidal A (1988) Characterization of ependymal cells in hypothalamic and choroidal primary cultures. Neuroscience 24:993-1007

Gabrion JB, Herbuté S, Bouillé C, Maurel D, Kuchler-Bopp S, Laabich A, Delaunoy JP (1998) Ependymal and choroidal cells in culture: characterization and functional differentiation. Microsc Res Tech 41:124-157. doi:10.1002/(SICI)1097-0029(19980415)41:2<124:: AID-JEMT3>3.0.CO;2-U

García MA, Millán C, Balmaceda-Aguilera C, Castro T, Pastor P, Montecinos H, Reinicke K, Zúñiga F, Vera JC, Oñate SA, Nualart F (2003) Hypothalamic ependymal-glial cells express the glucose transporter GLUT2, a protein involved in glucose sensing. J Neurochem 86:709-724

Goodman T, Hajihosseini MK (2015) Hypothalamic tanycytes-masters and servants of metabolic, neuroendocrine, and neurogenic functions. Front Neurosci 9:387. doi:10.3389/fnins.2015.00387

Grondona JM, Granados-Durán P, Fernández-Llebrez P, López-Ávalos MD (2013) A simple method to obtain pure cultures of multiciliated ependymal cells from adult rodents. Histochem Cell Biol 139:205220. doi:10.1007/s00418-012-1008-2

Hild W, Takenaka T, Walker F (1965) Electrophysiological properties of ependymal cells from the mammalian brain in tissue culture. Exp Neurol 11:493-501

Jarvis CR, Andrew RD (1988) Correlated electrophysiology and morphology of the ependyma in rat hypothalamus. J Neurosci 8:36913702

Jordan FL, Rieke GK, Hughes BW, Thomas WE (1990) Morphological diversity of ependymal cells in tissue culture. Brain Res Bull 25: $159-163$

Laabich A, Sensenbrenner M, Delaunoy JP (1989) Monolayer cultures of ependymal cells on porous bottom dishes. A tool for transport studies across the brain cerebrospinal barrier. Neurosci Lett 103:157161

Lazcano I, Cabral A, Uribe RM, Jaimes-Hoy L, Perello M, Joseph-Bravo P, Sánchez-Jaramillo E, Charli J-L (2015) Fasting enhances pyroglutamyl peptidase II activity in tanycytes of the mediobasal hypothalamus of male adult rats. Endocrinology 156:2713-2723. doi:10.1210/en.2014-1885

Meister B, Hökfelt T, Tsuruo Y, Hemmings H, Ouimet C, Greengard P, Goldstein M (1988) DARPP-32, a dopamine- and cyclic AMPregulated phosphoprotein in tanycytes of the mediobasal hypothalamus: distribution and relation to dopamine and luteinizing hormone-releasing hormone neurons and other glial elements. Neuroscience 27:607-622

Ouimet CC, Miller PE, Hemmings HC, Walaas SI, Greengard P (1984) DARPP-32, a dopamine- and adenosine $3^{\prime}: 5^{\prime}$-monophosphate-regulated phosphoprotein enriched in dopamine-innervated brain regions. III. Immunocytochemical localization. J Neurosci 4:111-124

Paull WK, Martin H, Scott DE (1977) Scanning electron microscopy of the third ventricular floor of the rat. J Comp Neurol 175:301-310. doi:10.1002/cne.901750305

Perraud F, Besnard F, Sensenbrenner M, Labourdette G (1987) Thrombin is a potent mitogen for rat astroblasts but not for oligodendroblasts and neuroblasts in primary culture. Int J Dev Neurosci 5:181-188

Peruzzo B, Pastor FE, Blázquez JL, Schöbitz K, Peláez B, Amat P, Rodríguez EM (2000) A second look at the barriers of the medial basal hypothalamus. Exp Brain Res 132:10-26

Peruzzo B, Pastor FE, Blázquez JL, Amat P, Rodríguez EM (2004) Polarized endocytosis and transcytosis in the hypothalamic tanycytes of the rat. Cell Tissue Res 317:147-164. doi:10.1007/ s00441-004-0899-1

Prevot V, Cornea A, Mungenast A, Smiley G, Ojeda SR (2003) Activation of erbB-1 signaling in tanycytes of the median eminence stimulates transforming growth factor beta1 release via prostaglandin E2 production and induces cell plasticity. J Neurosci 23:1062210632

Rejman J, Oberle V, Zuhorn IS, Hoekstra D (2004) Size-dependent internalization of particles via the pathways of clathrin- and caveolaemediated endocytosis. Biochem J 377:159-169. doi:10.1042/ BJ20031253

Rice D, Barone S (2000) Critical periods of vulnerability for the developing nervous system: evidence from humans and animal models. Environ Health Perspect 108(Suppl 3):511-533

Rinne UK (1966) Ultrastructure of the median eminence of the rat. Z Zellforsch Mikrosk Anat 74:98-122

Robins SC, Stewart I, McNay DE, Taylor V, Giachino C, Goetz M, Ninkovic J, Briancon N, Maratos-Flier E, Flier JS, Kokoeva MV, 
Placzek M (2013) $\alpha$-Tanycytes of the adult hypothalamic third ventricle include distinct populations of FGF-responsive neural progenitors. Nat Commun 4:2049. doi:10.1038/ncomms3049

Rodríguez EM, González CB, Delannoy L (1979) Cellular organization of the lateral and postinfundibular regions of the median eminence in the rat. Cell Tissue Res 201:377-408

Rodríguez EM, Blázquez JL, Pastor FE, Peláez B, Peña P, Peruzzo B, Amat $P$ (2005) Hypothalamic tanycytes: a key component of brainendocrine interaction. Int Rev Cytol 247:89-164. doi:10.1016/ S0074-7696(05)47003-5

Rodríguez EM, Blázquez JL, Guerra M (2010) The design of barriers in the hypothalamus allows the median eminence and the arcuate nucleus to enjoy private milieus: the former opens to the portal blood and the latter to the cerebrospinal fluid. Peptides 31:757-776. doi: 10.1016/j.peptides.2010.01.003

Smith PJ, Giroud M, Wiggins HL, Gower F, Thorley JA, Stolpe B, Mazzolini J, Dyson RJ, Rappoport JZ (2012) Cellular entry of nanoparticles via serum sensitive clathrin-mediated endocytosis, and plasma membrane permeabilization. Int J Nanomedicine 7:20452055. doi:10.2147/IJN.S29334

Tritschler F, Murín R, Birk B, Berger J, Rapp M, Hamprecht B, Verleysdonk $S$ (2007) Thrombin causes the enrichment of rat brain primary cultures with ependymal cells via protease-activated receptor 1. Neurochem Res 32:1028-1035. doi:10.1007/s11064-0069267-8

Weibel M, Pettmann B, Daune G, Labourdette G, Sensenbrenner M (1984) Chemically defined medium for rat astroglial cells in primary culture. Int J Dev Neurosci 2:355-366. doi:10.1016/0736-5748(84) 90072-8

Weibel M, Pettmann B, Artault JC, Sensenbrenner M, Labourdette G (1986) Primary culture of rat ependymal cells in serum-free defined medium. Brain Res 390:199-209

Wittkowski W (1998) Tanycytes and pituicytes: morphological and functional aspects of neuroglial interaction. Microsc Res Tech 41:29-42. doi:10.1002/(SICI)1097-0029(19980401)41:1<29::AIDJEMT4>3.0.CO;2-P 